\title{
RESPUESTAS DISPOSICIONALISTAS AL PROBLEMA WITTGENSTEINIANO-KRIPKEANO SOBRE EL SIGNIFICADO Y EL SEGUIMIENTO DE REGLAS
}

\author{
MANuel PÉrez Otero \\ Departamento de Filosofía \\ Facultad de Filosofía \\ Universidad de Barcelona \\ perez.otero@ub.edu
}

RESUMEN: Kripke formula cuatro objeciones en contra de una solución disposicionalista simple al enigma wittgensteiniano sobre seguir una regla. En este trabajo presento dos propuestas parcialmente disposicionalistas diferentes a la teoría disposicionalista simple que Kripke discute y defiendo que aquellas cuatro objeciones no les afectan. Una de esas dos propuestas puede atribuirse, con cierta precaución, al propio Wittgenstein. La otra, que me parece preferible, invoca una noción teleológica de disposición. Ambas propuestas apelan al concepto de simplicidad, o - alternativamente - al concepto de similitud natural. Rechazo también ciertas críticas que Kripke hace del uso de tales conceptos para solucionar el enigma sobre seguir una regla.

PALABRAS CLAVE: teleología, simplicidad, normatividad, intencionalidad, justificación racional

SUMMARY: Kripke formulates four objections to a simple dispositional solution to the Wittgenstenian rule-following puzzle. I present here two partially dispositional proposals, which are different from the simple view discussed by Kripke, and argue that they are not affected by his four objections to it. One of the two proposals may be attributed, although precautiously, to Wittgenstein himself. The other one, which I prefer, invokes a teleological notion of disposition. The two proposals rely on the concept of simplicity, or - alternatively - the concept of natural similarity. Some Kripkean criticisms of the use of such concepts to solve the rule-following problem are rejected.

KEY WORDS: teleology, simplicity, normativity, intentionality, rational justification

\section{Introducción}

En esta contribución me propongo explorar dos soluciones al problema sobre seguir una regla planteado en algunos textos del segundo Wittgenstein, particularmente en sus Investigaciones filosóficas (1953). Se trata de soluciones que, con algunas reticencias, podemos llamar disposicionales o disposicionalistas. Defenderé, específicamente, una respuesta disposicional-teleológica al problema.

Es pertinente concretar más el ámbito de mi exploración. En su análisis de esta problemática, Kripke (1982), tras presentar el enigma y descartar algunas otras posibles respuestas que Wittgenstein 
rechazaría, dedica unas quince páginas a comentar una respuesta disposicionalista. Contra esa respuesta elabora cuatro objeciones. Aunque Kripke no es partidario de la posición escéptica que atribuye a Wittgenstein, usualmente parece aceptar las críticas que ofrece a las diferentes soluciones posibles que va examinando (críticas que supuestamente Wittgenstein estaría también en condiciones de respaldar, conforme a la reconstrucción de Kripke). Así sucede en el caso de la solución disposicionalista. Mi objetivo es mostrar por qué la propuesta disposicional-teleológica que aquí planteo evita - a mi parecer- esas objeciones. Esto no implica que tales objeciones de Kripke sean incorrectas, pues se dirigen contra una solución emparentada aunque no exactamente coincidente con la hipótesis que propongo.

La finalidad de este texto no es principalmente exegética; sólo secundariamente haré referencias a lo que Wittgenstein pensaba realmente sobre las cuestiones aquí examinadas. No obstante, mencionaré también una solución meramente disposicionalista (que no incorpora el carácter teleológico) que - contra la opinión de Kripke y de muchos wittgensteinianos - podría atribuirse - aunque con bastantes matices - a Wittgenstein. Ese tipo de solución es algo más cercana a la que Kripke analiza y critica. También respecto a ella exploraré en qué medida se le aplican o no las objeciones de Kripke.

He mencionado posibles reticencias ante la opción de llamar disposicionales a esas soluciones. La razón es que el concepto de disposición (o el de disposición teleológica) no es el único concepto que desempeña en ellas un papel crucial. Será asimismo fundamental invocar la noción de simplicidad. Precisamente, Kripke también combate - en su libro - la idea de que conceptos como simplicidad o semejanza natural nos ayuden en el problema sobre seguir una regla. En la sección final me propongo contrarrestar las críticas de Kripke respecto a esta cuestión. Hechas estas consideraciones, seguiré refiriéndome a las soluciones aquí examinadas como disposicionales (aunque su denominación más apropiada quizá debería incorporar también referencia al concepto de simplicidad).

\section{La problemática sobre seguir una regla}

¿En qué consiste seguir una determinada regla? Supongamos que un sujeto, S, aparentemente aplica la regla de la suma a dos números ofreciendo como resultado de esa operación el número $r$. Nos preguntamos entonces por los factores que determinan que $\mathrm{S}$ está siguiendo la regla de la suma al decir que la suma de ambos números es $r$. 
Cabría contemplar dos alternativas: que $\mathrm{S}$ estuviera siguiendo otra regla, o que no estuviera siguiendo regla alguna. ¿En virtud de qué hechos S está siguiendo la regla de sumar? ¿Qué hechos fijan o determinan que está sumando? Habría muchas maneras de mostrar que esta indagación, cuando se comprende adecuadamente, no es trivial ni tiene una respuesta sencilla.

Obviamente existen números naturales más grandes que cualquier número natural que jamás haya sido sumado con otro. Para simplificar la representación gráfica del asunto, imaginemos que 56 es el mayor número jamás sumado (deberá ser claro que nada crucial depende de esta simplificación). Al sujeto $\mathrm{S}$ se le plantea entonces cuál es el resultado de sumar 57 con, por ejemplo, 68; es decir, cuál es el resultado de la operación $57+68$. Asumiendo que $\mathrm{S}$ ofrece como respuesta 125, ¿por qué es ésa una respuesta correcta? Definamos la parasuma como aquella operación matemática binaria sobre enteros positivos que aplicada a números menores de 57 tiene como resultado el mismo que la suma y en el resto de casos (es decir, cuando alguno de los dos números parasumados es mayor de 56) tiene siempre como resultado 5. Cada vez que en el pasado (cuando se encontró con números menores de 56) $\mathrm{S}$ - y el resto de la gente- sumó dos números, su conducta era compatible con la posibilidad alternativa de que S los hubiera parasumado, en lugar de sumarlos. Si S estuvo sumando, la respuesta que debería dar cuando se le pregunta por el resultado de $57+68$ es 125 . Si S estuvo parasumando, la respuesta correcta debería ser 5 . ¿Qué hechos determinan que hasta ahora $\mathrm{S}$ estuvo aplicando la regla de la suma y no la regla de la parasuma? ¿En virtud de qué el resultado correcto ofrecido por $\mathrm{S}$ debería ser 125 y no 5 ?

Hay una respuesta inmediata a esas cuestiones que probablemente sea verdadera. Pero no permitirá resolver el enigma, sino meramente reformularlo de una manera ligeramente diferente. Existe una relación representacional-intencional entre, por un lado, los usos de signos como "suma", "+" y todos sus derivados por parte de S y, por el otro, la operación abstracta de sumar. Una relación que no se da entre tales usos previos y la operación abstracta de parasumar. Digamos que $\mathrm{S}$, al usar esos signos, tenía intención de referirse a la suma; S quería decir que sumaba, no que parasumaba; S hacía referencia a la suma.

El núcleo principal de la cuestión original se replantea entonces bajo esta otra forma: ¿En virtud de qué hechos $\mathrm{S}$ quiso decir, o se refería a, la suma cada vez que usaba signos como "suma", "+". 
etc.? ¿Qué factores, dentro o fuera de su mente, han fijado ese significado para tales signos (fijando así la distinción entre respuestas correctas e incorrectas ante el requerimiento de aplicar la operación correspondiente también a pares de números nunca encontrados anteriormente)?

Esa última formulación del problema es la que mayormente adopta Kripke en su libro. Le seguiré a ese respecto, con objeto de visualizar mejor la solución disposicional-teleológica en tanto que solución inmune (según pretendo) a las críticas de Kripke a la respuesta disposicional más simple.

\section{Las objeciones de Kripke a la solución disposicional simple}

Con anterioridad a la difusión de las ideas del segundo Wittgenstein habría sido frecuente intentar solucionar el enigma planteado invocando aspectos subjetivos y/o fenomenológicos de la vida mental de los sujetos. No es necesario detenerse aquí en las poderosas críticas elaboradas por Wittgenstein contra estrategias de ese tipo, hoy suficientemente conocidas. Nuestro tema es la viabilidad de otro tipo de propuestas, que - con mayor o menor propiedad - pueden clasificarse como disposicionalistas.

Kripke señala que una solución disposicional simple sugerida ocasionalmente por algunos filósofos consistiría en lo siguiente:

(DS): S quiere decir la suma al usar signos como "más", "suma" y "+" si y sólo si S tiene la disposición a responder ante la pregunta por cualquier suma " $x+y$ " indicando cuál es, efectivamente, la suma de $x$ e $y$.

Análogamente, S querría decir la parasuma al usar signos como "más", "suma" y "+" si y sólo si S tuviera la disposición a responder, ante la pregunta por cualquier operación " $x+y$ " indicando la parasuma de $x$ e $y$ (cfr. Kripke 1982, pp. 22-23).

Pero esa teoría disposicional simple sobre la fundamentación de nuestro seguimiento de reglas es incorrecta, según Kripke. Para mostrarlo enumera cuatro dificultades.

La primera es el problema de la justificación racional. Kripke considera que en el planteamiento wittgensteiniano de este enigma se incluye la idea de que los factores en virtud de los cuales $\mathrm{S}$ sigue una regla al realizar cierta acción deberían proporcionar a $\mathrm{S}$ una justificación racional (una razón justificativa) de que es así cómo se ha de actuar al aplicar la regla; $\mathrm{S}$ debe ser guiado racionalmente por 
tales factores. Según Kripke, la disposición de $\mathrm{S}$ a contestar de ciertos modos ante preguntas sobre sumas le compele a dar determinadas respuestas, pero no le justifica en absoluto; $\mathrm{S}$ no puede hallar en su disposición una razón, una guía, para ofrecer las respuestas que ofrece.

Kripke presenta luego como si fuera una especie de reformulación de esa primera dificultad lo que en realidad es un problema algo diferente. Concierne a lo que suele denominarse normatividad: debe existir una distinción entre aplicaciones correctas y aplicaciones incorrectas de una regla. ${ }^{l}$ No puede ocurrir que cualquier respuesta que a $\mathrm{S}$ le parezca correcta sea necesariamente correcta. Y precisamente la propuesta disposicionalista simple estaría incapacitada para trazar esa distinción correcto/incorrecto. Conforme a esa propuesta, lo que S está dispuesto a responder ante una pregunta por la suma (y, suponemos, le parece correcto responder) coincide con la respuesta correcta. Según Kripke, "casi todas las objeciones a la concepción disposicional se reducen" a esta doble objeción (el problema de la justificación racional y el problema de la normatividad), que él tiende a unificar (cfr. Kripke 1982, p. 24.)

Otro inconveniente es el problema de la finitud. En varios sentidos, los humanos somos seres finitos. No sólo respecto a nuestras actuaciones reales, sino también respecto a nuestras disposiciones potenciales (que fijarían nuestras acciones potenciales). Cuando se le pregunte a $\mathrm{S}$ por la adición de ciertos números muy grandes, no es cierto que $\mathrm{S}$ tendrá la disposición de responder indicando cuánto suman. En muchos casos S morirá de viejo antes de tener tiempo para dar una respuesta.

El último problema presentado por Kripke deriva de las disposiciones que podamos tener a cometer errores (este inconveniente podría verse como una versión particular del problema de la normatividad). El sujeto, S, quizá tenga una tendencia a equivocarse sistemáticamente al aplicar algún algoritmo para sumar. Por ejemplo, supongamos que cuando $\mathrm{S}$ escribe dos números grandes, uno debajo del otro, y procede a sumarlos conforme al algoritmo familiar que solemos aprender de niños, generalmente olvida llevarse una en los casos que corresponde. No tiene entonces la disposición a responder

${ }^{1}$ Sería más apropiado referirse a ese fenómeno empleando algún otro término; un candidato podría ser evaluabilidad. La expresión "normatividad" se vincula con "norma". Pero no siempre que cabe aplicar la distinción correcto/incorrecto hay normas involucradas; no parece haberlas, por ejemplo, en casos de funcionamiento apropiado o anómalo de una función biológica. 
adecuadamente indicando la suma; tiene una disposición a errar en tales circunstancias.

Tal y como sucedía con los dos problemas anteriores (el problema de la normatividad y el problema de la finitud), para entender esta última dificultad hemos de asumir que el sujeto en cuestión, S, se refiere a la suma al usar los signos relevantes pese a que a veces, por algún error que tiende a cometer (o por algún error ocasional, o porque los números sean demasiado altos), no tiene la disposición a indicar cuál es la suma de los números por los cuales se le pregunta. No se trata de un sujeto cuya conducta o competencia fuera tan aberrante que no hubiera aprendido el significado de "suma", "+" y sus derivados (de modo que nunca se refiriese con ellos a la suma), pues entonces no tendríamos contraejemplos a la teoría disposicional. Se presupone que $\mathrm{S}$ quiso decir la suma (no la parasuma, ni ninguna otra operación diferente), aunque sus disposiciones no siempre le llevan a dar la respuesta correcta.

\section{Una propuesta disposicional menos simple}

Wittgenstein se manifestó contrario a las concepciones disposicionales sobre los estados mentales. Aparentemente, tener intención de referirse a la suma no sería, para él, estar en un estado disposicional (cfr. Investigaciones filosóficas, § 149; Zettel, § 610). Sin embargo, tal y como he defendido en Pérez Otero 2001, siguiendo parcialmente a García-Carpintero (1996, sec. XI.3), sus declaraciones al respecto son compatibles con atribuirle una posición disposicional deflacionaria, por decirlo así. Veamos por qué.

Las disposiciones, o propiedades disposicionales, pueden interpretarse conforme a dos concepciones. Para cada disposición que podamos reconocer hay asociada una proposición expresable mediante un enunciado que contiene como parte especialmente relevante una sub-fórmula condicional subjuntiva. Por ejemplo, una posible representación de la proposición asociada con la solubilidad (un caso paradigmático de propiedad disposicional) vendría dada por el siguiente enunciado: "Para todo $x, x$ es soluble si y sólo si [en condiciones normales, si $x$ se introdujera en agua, entonces $x$ se disolvería]". Una visión realista de las disposiciones tiende a considerar que tener la disposición es tener una propiedad estructural interna específica (común para todos los individuos que tienen la disposición, según muchas versiones de esta idea) de la que depende causalmente que se satisfaga el condicional subjuntivo correspondiente (la parte correspondiente a "en condiciones normales, si ocurriera C, entonces ocu- 
rriría R"). Una visión deflacionaria, de inspiración humeana, puede aceptar la existencia de disposiciones pero sosteniendo que tener una disposición es meramente satisfacer dicho condicional subjuntivo.

Wittgenstein combatía la idea de que un estado mental (una creencia, un dolor, una intención) se identificara conceptualmente, en última instancia, con tener una propiedad estructural neurológica, o física, común para cualquier sujeto al que se le atribuyera correctamente dicho estado mental. Pero su visión del asunto es cercana a las posiciones en filosofía de la mente que luego se conocieron como funcionalistas: es irrelevante cuál sea la base física que realiza un estado mental, pues seres de especies biológicas muy diferentes (humanos y marcianos, por ejemplo) podrían diferir enormemente respecto a tales bases físicas; lo crucial, en la identificación de un estado mental, es la apropiada correlación entre inputs y outputs. Y ese programa funcionalista aspira a caracterizar esas correlaciones mediante condicionales subjuntivos que, simplificando, son del tipo indicado: "en condiciones normales, si ocurriera C, entonces ocurriría R". En definitiva, me parece acertado atribuir a Wittgenstein una suerte de concepción disposicionalista deflacionaria sobre nuestra vida mental. (Una discusión a fondo sobre cuál es la correcta interpretación de Wittgenstein respecto a este asunto excede con mucho los límites de este artículo, cuyo objetivo no es prioritariamente exegético.)

Volvamos ahora al enigma sobre seguir una regla, ilustrado con el caso del sujeto $\mathrm{S}$ y la regla de la suma. Propongo caracterizar el modelo de solución disposicional que Wittgenstein podría sostener (dejando ahora de lado su animadversión a la teorización explícita precisa) con esta formulación:

(DW): Si

(i) en condiciones normales, si se preguntara a $\mathrm{S}$ por la suma " $x$ $+y^{\prime \prime}$, en los casos básicos $\mathrm{S}$ se sentiría inclinado a responder indicando cuál es, efectivamente, la suma de $x$ e $y$,

(ii) no es el caso que $\mathrm{S}$ crea que la operación por la que se le pregunta está definida sólo para un subconjunto propio de todos los números naturales, y

(iii) la suma es la operación matemática más simple que extiende a los restantes casos (para el resto de pares de números naturales) esa disposición de $\mathrm{S}$, 
entonces S quiere decir la suma al usar signos como "más", "suma" y "+". 2

En esa propuesta intervienen cuatro elementos que no estaban presentes en la concepción disposicional simple que Kripke discute. Dos de ellos son cruciales para proteger a esta solución de las objeciones de Kripke a la solución disposicional simple (tal y como intento defender en las secciones 4 y 6). Se trata de la apelación a los conceptos de casos básicos y de simplicidad. Invocar cierta noción de condiciones normales no es tan importante a la hora de evitar esas objeciones. El otro elemento adicional, la cláusula (ii), me parece necesario porque sin esta cláusula el análisis sería incorrecto por contraejemplos como el siguiente. Sea la minisuma una operación binaria definida sólo sobre los primeros $10^{99}$ números naturales, que aplicada a cualesquiera dos de ellos arroja el mismo resultado que la suma. Supongamos que a $\mathrm{S}$ se le hubiera instruido en el uso de signos como "más", "suma", "+", etc., indicándole explícitamente que hacen referencia a (lo que aquí he llamado) la minisuma. Es decir, $\mathrm{S}$ sabe que la operación en cuestión sólo se aplica a un número finito de casos. En estas circunstancias, $\mathrm{S}$ no se estaría refiriendo a la suma al usar tales signos, sino a la minisuma. Pero es razonable pensar que se cumpliría el antecedente de (DW) si en el mismo no constara la cláusula (ii).

Los cuatro elementos aparecerán también (con análogos propósitos) en la propuesta disposicional-teleológica que exploro y defiendo en la sección 5. Dejaré para la última sección (sec. 6) la discusión sobre cómo la condición sobre la simplicidad evitaría el problema de la finitud. En la próxima sección evalúo en qué medida las otras tres críticas de Kripke se aplican también a (DW). Se verá que gran parte de la discusión es relevante también respecto a la solución disposicional-teleológica.

\section{Justificación racional, normatividad y diversidad de respuestas}

No es necesario invocar alguna de esas diferencias entre la propuesta disposicional wittgensteiniana, (DW), y la propuesta disposicional simple, (DS), para afrontar el problema de la justificación racional planteado por Kripke. El requerimiento de que los factores

${ }^{2}$ (DW) es un enunciado condicional, no un bicondicional como (DS). No quiero descartar que $\mathrm{S}$ pueda referirse a la suma sin saber sumar. Por ejemplo usando "suma" en determinadas circunstancias (análogamente a cómo puede alguien referirse al agua usando "agua" en determinadas circunstancias, sabiendo muy poco sobre el agua). La discusión, de todos modos, es sobre un sujeto que sabe sumar. 
determinantes de que $\mathrm{S}$ esté siguiendo una regla proporcionen a $\mathrm{S}$ una razón justificativa para sus acciones nos compromete con una posición internista-intelectualista epistemológica, ajena a las convicciones naturalistas de Wittgenstein. Para él, la búsqueda de razones no se prolongaría indefinidamente:

[...] hay una idea de que "algo tiene que hacernos hacer lo que hacemos". Y esto vuelve a enlazarse con la confusión entre causa y razón. No necesitamos tener razón alguna para seguir la regla tal como lo hacemos. La cadena de razones tiene un límite. (1958, p. 183)

“¿Cómo puedo seguir una regla?” - si ésta no es una pregunta por las causas, entonces lo es por la justificación de que actúe así siguiéndola.

Si he agotado los fundamentos, he llegado a roca dura y mi pala se retuerce. Estoy entonces inclinado a decir: "Así simplemente es cómo actúo." (1953, § 217)

Eso no significa que Kripke cometa un error mayúsculo al plantear ese requerimiento internista-intelectualista en su reconstrucción del enigma que atribuye a Wittgenstein. El error que le atribuyo no es tan notorio. Kripke intenta reconstruir lo que considera que es una posición wittgensteiniana escéptica (respecto a la objetividad fáctica de nuestro seguimiento de reglas: según Kripke, Wittgenstein considera que no hay hechos objetivos en virtud de los cuales es verdad que alguien sigue una regla al usar signos con cierto significado). El requerimiento de justificación racional parece muy plausible: es sensato suponer que los presuntos hechos que supuestamente determinan qué quiso decir un sujeto deben ser capaces de guiar al sujeto en sus aplicaciones del signo. Así pues, es legítimo contemplar versiones de la problemática sobre seguimiento de reglas que incluyan ese requerimiento como parte sustancial del problema. Sin embargo, el requisito internista-intelectualista derivaría de ideas filosóficas tradicionales que Wittgenstein rechaza; no se requiere - según Wittgenstein - que algo tenga que hacernos hacer lo que hacemos, si ese algo se concibe como una razón que nos guíe. Y es un requisito que un externista epistemológico también puede legítimamente rechazar (basándose en consideraciones no necesariamente derivadas de los textos de Wittgenstein). Acierta Kripke al atribuir a Wittgenstein el rechazo de cualquier solución al enigma que mantenga esa condición internista-intelectualista. Pero, en mi opinión, se equivoca al suponer que eso es dialécticamente adecuado en su descripción del espacio lógico disponible para Wittgenstein. Porque la descripción 
kripkeana presupone que, según Wittgenstein, sin el requisito epistémico internista-intelectualista no puede haber objetividad fáctica sobre el significado en nuestro seguimiento de reglas. Pero Wittgenstein puede sostener una posición fáctica sobre el significado (una posición no escéptica respecto a la objetividad fáctica) pero no internista, como la que — con matices - le atribuyo cuando afirmo que (DW) encajaría en su concepción. En definitiva, las reiteradas observaciones antiintelectualistas de Wittgenstein sobre nuestra justificación (contrarias al requisito epistémico internista) serían elementos a favor de una concepción epistemológica externista sobre las reglas, no necesariamente elementos a favor de una concepción contraria a la objetividad fáctica sobre el significado. ${ }^{3}$

Puesto que mi investigación no es principalmente exegética, lo que aquí más importa es saber si podemos o no prescindir del requerimiento de justificación racional. Pero no es factible proseguir ese debate en este ensayo. Tanto (DW) como mi propuesta disposicionalteleológica de la próxima sección, (DT), prescinden del requisito internista-intelectualista. Su viabilidad depende de esta cuestión. En cualquier caso, el problema de la justificación racional que Kripke plantea puede ser menos grave de lo que él parece asumir.

Reconozco que la parte más controvertida de la hipótesis (DW) - así sucede también con (DT) - es su supuesta capacidad para hacer frente al problema de la normatividad y al problema de las disposiciones a errar invocando la noción de casos básicos y también parcialmente la noción de simplicidad (la simplicidad intervendrá, sobre todo, para evitar el problema de la finitud). Lo que diré será muy tentativo y esquemático. También en esto un análisis detallado requeriría mucha mayor extensión.

Cualquier teoría sobre la naturaleza de las disposiciones debe poder explicar la existencia de excepciones: casos en que un objeto tiene

${ }^{3}$ El enigma sobre seguir una regla es, en cualquier caso, un problema importante y de muy difícil resolución. Pero resulta especialmente problemático o paradójico desde perspectivas epistemológicas fuertemente internistas como las de Wright (1984) o Boghossian (1989). Algunas reflexiones cruciales de Wittgenstein contra un requisito internista-intelectualista de ese tipo se encuentran sobre todo en pasajes del Cuaderno Marrón (cfr., especialmente, pp. 123-124 y 183), y pueden reconstruirse en forma de una sólida argumentación contra la necesidad de tales símbolos-guía en la comprensión del significado (diferente a la argumentación contra la suficiencia de símbolos-guía en la comprensión del significado, derivada del grueso de sus consideraciones sobre seguir una regla). A mi juicio, la epistemología internista no ha atendido suficientemente a esas reflexiones wittgensteinianas, que quizá no ha sabido discernir con claridad en el marco general de la discusión sobre seguimiento de reglas. He analizado esa argumentación en Pérez Otero 2000. 
la disposición pero no satisface el condicional subjuntivo. También cuando no hay normatividad involucrada, como sucede con la solubilidad. Es decir, ilustrado con ese caso, debe aceptarse que algunos objetos son solubles incluso si no se disolvieran si se les introdujera en agua. La cláusula de condiciones normales está destinada a paliar esta dificultad (o alguna claúsula similar de condiciones parejas, ceteris paribus: condiciones similares a aquellas en que típicamente se manifiesta la disposición): si el objeto no se disolviera sería porque las condiciones no serían normales. Para la problemática que nos ocupa no parece que dicha cláusula pueda ayudar mucho. Promete mayor utilidad la cláusula sobre casos básicos. La idea es que para cualquier sujeto que aprende a sumar habrá casos paradigmáticos de aplicación de la operación en los cuales no se equivoca. La hipótesis de que no existen tales casos para cierto sujeto crece simultáneamente con la hipótesis de que no ha aprendido realmente la suma. Y es con respecto a tales casos básicos, o paradigmáticos, que se define la manifestación de la disposición (responder a la pregunta por la suma indicando realmente la suma). Conforme nos alejamos de ese tipo de casos se acrecienta la posibilidad de error (sea o no sistemático). Pero se evita el problema de la normatividad y al problema de las disposiciones a errar porque, a diferencia de lo que sucedía con la propuesta disposicional simple, (DS), la hipótesis (DW) no implica que cualquiera que fuera la respuesta de $\mathrm{S}$ dicha respuesta coincidirá con la operación suma a la que se refiere $\mathrm{S}$ cuando usa los signos correspondientes. Si S ha aprendido a sumar y ha aprendido el vocabulario correspondiente (el uso de signos como "suma", "más", etc.), entonces quiere decir la suma aunque su respuesta ante algunos casos no básicos quizá no coincida con aquello a lo que se refiere.

$\mathrm{Si}$ sus disposiciones a responder sólo permiten fijar el significado de "suma" y los otros signos usados por $\mathrm{S}$ respecto a los casos básicos, ¿cómo se fija el significado de "suma" respecto a los otros casos? Conforme a la cláusula (iii), la simplicidad se encarga de ello ( $c f r$. la sección 6).

Una idea subyacente a esta propuesta es la siguiente. Aunque cabe trazar una distinción entre aplicaciones correctas y aplicaciones incorrectas de la regla, eso sólo es factible en la medida en que nos alejamos de los casos básicos o paradigmáticos. Para estos últimos, en cierto modo, la inclinación natural a reaccionar de cierta manera coincidirá con la corrección en la aplicación de la regla. (Tenemos intuiciones favorables a esa posición. Descartamos que desde los primeros usos del lenguaje un bebé pueda estar usando palabras ya con 
el mismo significado que nosotros, pero aplicándolas incorrectamente. $)^{4}$

Hay riesgo de interpretar eso de forma inapropiada. Sería un inconveniente fatal para cualquier teoría que de ella se siguieran enunciados condicionales contrafácticos como éstos:

(*) Si [un sujeto, S* (quizá un extraterrestre), tuviera una naturaleza biológica tan peculiar que se sintiera inclinado a reaccionar incluso en casos básicos de forma muy diferente a la nuestra, indicando, por ejemplo, ante la pregunta por la suma de 3 y 2 , que el resultado es 7], entonces 3 y 2 sumarían 7 (o sumarían 7 para $S$, o según $S$ ).

(**) Si [nosotros tuviéramos una naturaleza biológica tan diferente a la que de hecho tenemos que nos sintiéramos inclinados a reaccionar incluso en casos básicos de forma muy diferente a la nuestra, indicando, por ejemplo, ante la pregunta por la suma de 3 y 2 , que el resultado es 7], entonces 3 y 2 sumarían 7 .

Pero la hipótesis (DW) no implica enunciados condicionales de ese tipo. Una cuestión secundaria es que tal vez sea imposible (metafísicamente imposible; quizá también conceptualmente imposible) la existencia de sujetos como los descritos en $\left({ }^{*}\right)$ y, sobre todo, en $\left({ }^{* *}\right)$. La cuestión fundamental es que aunque existiera un sujeto, $\mathrm{S}^{*}$, con los rasgos descritos en el antecedente de $(*)$, no es legítimo concluir -invocando (DW) - lo dicho por su consecuente. Si la desviación de $\mathrm{S}^{*}$ en las respuestas correctas no es por la relativa complicación de los casos comparativamente menos básicos, sino que ya en los casos básicos siente una inclinación diferente a la que sentimos nosotros, entonces la operación a la que $S^{*}$ se referiría con la expresión "su$m a$ " aplicada a 3 y 2 tendría como resultado 7 . Pero su signo "suma" no significaría lo mismo que el nuestro. Así, no sería propiamente una aplicación incorrecta de la regla de la suma, sino una aplicación correcta de otra regla. Ocasionalmente, alguien puede describir la situación diciendo que $\mathrm{S}^{*}$ no aplica la regla correctamente. Pero es crucial entender la diferencia entre este extraterrestre y un sujeto que comparte nuestras inclinaciones básicas pero se equivoca ante

\footnotetext{
${ }^{4}$ Cfr. Pérez Otero 2000. La distinción entre casos básicos, o paradigmáticos, y los casos restantes se correlaciona con la distinción entre lo que Wittgenstein denomina, respectivamente, juicios y opiniones.
} 
una suma complicada. La propuesta (DW) dispone de bases teóricas para distinguirlos. Para el caso de $(* *)$ la situación es análoga. ${ }^{5}$

Como es obvio, esta concepción hace recaer un gran peso en la noción de caso básico. Sería muy deseable una mayor elucidación de esa noción. Una tarea que excede con mucho los límites (también de espacio) de esta investigación. Sólo añadiré aquí tres comentarios: (a) No constituye un defecto de $(\mathrm{DW})$ el hecho de que la separación entre casos básicos y los otros casos sea gradual. Llamar la atención hacia la vaguedad en nuestras representaciones del mundo, o en el mundo mismo, es otro de los numerosos méritos de Wittgenstein. ${ }^{6}$ (b) En la última sección señalaré la conveniencia de aceptar que el concepto de caso básico debe relativizarse a sujetos o a tipos de sujetos (dependiendo de las disposiciones de los mismos), para que - en combinación con el concepto de simplicidad - pueda contestarse a una posible objeción. (c) Esa relativización de los casos básicos (a sujetos, o a tipos de sujetos) no implica que la distinción entre casos básicos y no básicos carezca de base objetiva. Según creo, la distinción depende de nuestras formas de ser (derivadas de la naturaleza innata de los sujetos combinada con su naturaleza adquirida). Pero muy probablemente el concepto mismo de caso básico sólo pueda explicarse mediante ilustraciones, sin poder ser caracterizado de forma no circular.

\section{La hipótesis disposicional-teleológica}

La discusión de la sección anterior sugiere que la hipótesis disposicional wittgensteiniana, (DW), está en la senda correcta, al menos para evitar las objeciones de Kripke a la hipótesis disposicional simple, (DS). Me parece preferible, sin embargo, una hipótesis que difiere de (DW) en su primera cláusula, (i). Esta otra hipótesis se acerca a las interpretaciones realistas de las disposiciones, aunque añade también un elemento adicional: un componente funcional-teleológico. Podría formularse así:

(DT): Si

${ }^{5}$ Los lectores familiarizados con los experimentos mentales sobre la Tierra Gemela pueden hallar ilustrativa la siguiente comparación. El contrafáctico $\left({ }^{* *}\right)$ es erróneo de forma similar a como sería erróneo este contrafáctico: si nosotros hubiéramos vivido siempre en la Tierra Gemela, entonces el agua no habría sido $\mathrm{H}_{2} \mathrm{O}$.

${ }^{6}$ En Pérez Otero (2016) sostengo que Wittgenstein presenta datos intuitivos favorables al pluralismo sobre el seguimiento de reglas (la imposibilidad de que hubiera un único caso de seguirse alguna regla) derivados de la vaguedad en el proceso de aprendizaje de reglas. (Eso es compatible con que dicho pluralismo también derive del comunitarismo.) 
(i) en condiciones normales, $\mathrm{S}$ tiene la disposición teleológica a responder ante la pregunta por la suma " $x+y$ " en los casos básicos indicando cuál es, efectivamente, la suma de $x$ e $y$,

(ii) no es el caso que $\mathrm{S}$ crea que la operación por la que se le pregunta está definida sólo para un subconjunto propio de todos los números naturales, $\mathrm{y}$

(iii) la suma es la operación matemática más simple que extiende a los restantes casos (para el resto de pares de números naturales) esa disposición de $\mathrm{S}$,

entonces S quiere decir la suma al usar signos como "más", "suma" y "+".

Debo clarificar en qué sentido hablo aquí de disposición teleológica. Me refiero en realidad a lo que frecuentemente se describe como una función, aunque también bajo ciertos supuestos respecto a dicho concepto, en particular el supuesto de incorporar el carácter teleológico o aparentemente teleológico. En ese sentido, tener la función de hacer (o de dar como resultado) $\mathrm{R}$ es, en líneas generales, tener una disposición a hacer $\mathrm{R}$ que se tiene para hacer $\mathrm{R}$, una disposición cuyo fin es hacer R. (Enseguida ampliaré esa idea.) Dicho rasgo es distintivo sólo de algunas disposiciones. Por ejemplo, no parece haber teleología involucrada en casos paradigmáticos de disposiciones como la solubilidad o la elasticidad: en la disposición a disolverse de un objeto no hay fines, el objeto no tiene la propiedad disposicional para disolverse.

Hay diferentes concepciones filosóficas sobre las funciones teleológicas. El enfoque etiológico propugnado por Larry Wright (cercano al que propuso posteriormente R.G. Millikan) es el que me parece menos problemático ( $c f r$. Wright 1973; 1976.) Entre las virtudes de su propuesta se incluye que intenta captar mediante su definición las características comunes a dos tipos de teleología: los casos en que hay intencionalidad involucrada (acciones, instituciones sociales, artefactos) y aquellos otros en que la finalidad puede explicarse mediante la evolución natural (las funciones biológicas) ( $c f r$. Pérez Otero 2003.)

Con objeto de clarificar nuestra comprensión de (DT), propongo la siguiente caracterización de las disposiciones, o funciones, teleológicas, inspirada en el análisis de Wright:

$\mathrm{X}$ tiene la disposición teleológica de hacer (o de dar como resultado) $\mathrm{R}$ si y sólo si 
(a) X tiene la disposición de hacer (o de dar como resultado) $\mathrm{R}$ y

(b) Hay una explicación causal de por qué $\mathrm{X}$ tiene esa disposición en la que desempeña un papel relevante la regularidad nómica consistente en que entidades físicamente como X (en particular, con esa misma disposición) pueden hacer (o dar como resultado) $\mathrm{R}$

Con frecuencia, la cláusula (b) queda más sintéticamente resumida mediante locuciones como "la disposición existe porque causa R". Se alude con ello a un rasgo típico y potencialmente sorprendente de la teleología: la causalidad final, con su apariencia de que, en cierta manera, el futuro afecta causalmente al pasado. Sin embargo, dicha cláusula (b) es perfectamente coherente con una concepción plenamente naturalista sobre la causalidad. Cuando la disposición es un estado o una propiedad adquirida a través del aprendizaje (como plausiblemente sucede con la disposición a responder apropiadamente preguntas sobre sumas) quizá no se visualiza tan fácilmente el cumplimiento de (b). Ilustrémoslo primero muy esquemáticamente con funciones biológicas y con artefactos. La disposición de un determinado corazón, $\mathrm{X}$, a bombear sangre (su función) es teleológica porque, efectivamente, la regularidad nómica consistente en que entidades físicamente como $\mathrm{X}$ podían bombear sangre ha sido relevante en la explicación causal de por qué existe $\mathrm{X}$ con esa misma disposición. Ha sido relevante en el modo descrito por la teoría de la selección natural. Se cumple, pues, (b). También se cumple (b) en casos de artefactos. La disposición de un determinado termómetro, Z, a medir la temperatura corporal (su función) es teleológica porque, efectivamente, la regularidad nómica consistente en que entidades físicamente como $\mathrm{Z}$ podían medir la temperatura corporal ha sido relevante en la explicación de por qué existe $Z$ con esa misma disposición. Ha sido relevante porque los diseñadores de $Z$ han tenido presente esa regularidad (sin necesidad, en este caso, de que previamente hubiera habido otros termómetros). Con las disposiciones adquiridas tras procesos de aprendizaje se cumpliría también (b): llega a estabilizarse y persistir una determinada disposición del sujeto $\mathrm{S}$ en parte como resultado de su apropiada manifestación (en forma de respuestas en los casos básicos) en las situaciones en que $\mathrm{S}$ estaba todavía aprendiendo la regla correspondiente.

En este texto restrinjo la discusión a cómo (DW) y ahora (DT) evitan o no las objeciones de Kripke a (DS). Mi principal motivación para propugnar (DT) en lugar de (DW) es que incluso si (DW) efectivamente evita esas objeciones, tiene otras dificultades que aconsejan 
— desde mi punto de vista - algo en la línea de (DT). Tales dificultades se resumirían esquemáticamente en los siguientes términos. Tener o no una mera disposición (no teleológica) depende sólo de rasgos intrínsecos del sujeto. Por el contrario, tener una disposición teleológica depende también de rasgos intrínsecos (pues la etiología de la disposición teleológica es relevante). Hay intuiciones muy arraigadas a favor de que el contenido de los estados mentales (como el de referirse a la suma) depende de rasgos extrínsecos a tales estados; particularmente de su etiología. Son intuiciones favorables al externismo intencional, que desde diferentes perspectivas han puesto de relieve el propio Wittgenstein así como Donald Davidson, Tyler Burge y posteriormente muchos otros. Derivan, principalmente, de intuiciones similares contrarias a que las propiedades semánticas de una representación dependan de sus rasgos intrínsecos. Quizá la mejor ilustración de esta última idea procede de Putnam, con ejemplos como éste: en determinadas circunstancias, la figura de una flecha pintada dentro de una cueva representa la salida; pero una figura visualmente indistinguible de ella (equivalente en sus rasgos intrínsecos) que se hubiera formado aleatoriamente como resultado de la humedad no representa nada ( $c f r$. Putnam 1981, cap. 1) ${ }^{7}$

De entre las diversas respuestas al enigma sobre seguir una regla (especialmente en la versión de Kripke), la que Millikan desarrolla (1990) es la más cercana a (DT). Ella también apela al concepto de función, basándose en un análisis etiológico del mismo. Precisamente, una diferencia importante entre (DT) y la propuesta de Millikan se relaciona con las ventajas que - a mi parecer - tiene el enfoque de L. Wright sobre las funciones teleológicas frente al de Millikan. (DT) no implica ni presupone que una disposición teleológica tenga rasgos relevantemente semejantes a las funciones biológicas, pese a que nuestro conocimiento empírico de la teoría de la evolución natural (independiente de nuestra captación general, más abstracta, del concepto general de función - en el sentido teleológico) nos permita suponer que - tal y como ya he indicado - nuestras disposiciones

${ }^{7} \mathrm{El}$ asunto también se ilustra en el experimento mental elaborado por Davidson (1987), sobre el Hombre de los Pantanos (una entidad microfísicamente indiscernible de un ser humano, pero que es el resultado aleotorio de la caída de un rayo sobre un tronco); aunque con una diferencia crucial respecto al ejemplo de Putnam: las intuiciones sobre el caso de Davidson están mucho más divididas. Basándome en el análisis etiológico de las funciones teleológicas, en Pérez Otero 1994 he desarrollado una noción de superveniencia apta para acomodar esa naturaleza extrínseca de los estados mentales en un marco naturalista, conforme a las tesis externistas sobre el contenido y, concretamente, coincidente con Davidson al rechazar la atribución de estados mentales al Hombre de los Pantanos. 
teleológicas innatas serán típicamente funciones biológicas. (En esto la solución de Millikan se aleja más de Wittgenstein; aunque ello no podría verse como una crítica, pues Millikan no pretende moverse cerca de parámetros wittgensteinianos.) Otras diferencias notables conciernen a mi invocación explícita —en (DT) y en la discusión de este artículo - del concepto de simplicidad ( $c f r$., en particular, la siguiente sección). Es cierto que la solución de Millikan también presupone ciertas distinciones ontológicas entre propiedades o regularidades naturales, por una parte, y propiedades o regularidades artificiosas o comparativamente menos naturales, por otra ( $c f r$. Millikan 1990 , p. 334). Pero no queda suficientemente claro — a mi juiciocómo la simplicidad y/o la naturalidad intervendrían exactamente en su respuesta al enigma, a diferencia de la articulación concreta recogida en (DT). Además, otro rasgo original de mi tratamiento es que voy a proponer (sección 6) respuestas específicas a cada una de las críticas formuladas por Kripke contra la idea de que la simplicidad o la semejanza natural puedan ayudarnos a resolver el problema.

¿Cómo afronta la hipótesis disposicional-teleológica, (DT), aquellas críticas de Kripke a la propuesta disposicional simple, (DS)? La discusión en la sección anterior es válida también ahora con respecto a (DT). Por una parte, el problema de la justificación se deja de lado con independencia de la viabilidad de una u otra propuesta disposicionalista. Por otra parte, aunque la cláusula (i) de (DT) no coincide exactamente con la correspondiente cláusula de (DW), el papel que cabe atribuir a la apelación a los casos básicos — combinada con la apelación a la simplicidad en la cláusula (iii) — no difiere sustancialmente del papel que ya he comentado en la sección precedente en relación con (DW). Creo pues que (DT) también está protegida ante el problema de la normatividad y el problema de las disposiciones a errar. En la siguiente sección veremos cómo estas propuestas afrontan el problema de la finitud. ${ }^{8}$

${ }^{8}$ Como consideración general, creo que las concepciones positivas de Wittgenstein sobre la mente y el significado habrían sido más sólidas si la teleología hubiera tenido mayor presencia en sus reflexiones. Eso concierne también al Tractatus. La tesis de que todo nombre genuino nombra alguna entidad (razonablemente derivable del supuesto de que el significado de un nombre es el portador del nombre) encuentra mayores dificultades (por ejemplo, ante la eventualidad de que no exista entidad presuntamente nombrada) que la tesis de que todo nombre genuino tiene como función teleológica nombrar alguna entidad. También ese neomillianismo teleológico insinuado tendría problemas (no es claro cómo podría distinguir el significado de "Sancho Panza" del significado de "Columbo"); pero no tan acuciantes como los problemas del millianismo defendido en el Tractatus. No hay ocasión aquí para explorar estas cuestiones. 


\section{Finitud y simplicidad}

La hipótesis disposicional simple, (DS), tenía el problema de la finitud porque nuestras disposiciones a sumar no involucran cualesquiera números; casi todos los números serán demasiado altos. La cláusula (iii) — tanto en la propuesta wittgensteiniana como en la teleológica - pretende solventar esta dificultad.

Mi apelación a la noción de simplicidad deriva principalmente de Lewis 1983. En ese artículo, Lewis expone por qué para resolver satisfactoriamente diversos problemas metafísicos parece imprescindible recurrir a algún concepto primitivo (no analizable reductivamente mediante otros conceptos) de naturalidad, o de simplicidad, o de semejanza natural. Entre esos problemas se incluyen algunos concernientes a la determinación del significado de nuestras representaciones; también —en particular - el enigma sobre seguir una regla.

La cláusula (iii) es todavía imprecisa, en varios sentidos. La he formulado usando el concepto de simplicidad, pero (siguiendo a Lewis) no tengo en mente una acepción suficientemente concreta como para distinguirlo del concepto de naturalidad. Incluso podría entenderse esa cláusula involucrando en su lugar la idea de semejanza natural. Se trataría entonces, aproximadamente, de lo siguiente: la suma es, de entre las diversas operaciones matemáticas definidas sobre todos los números naturales, la operación más semejante a las operaciones definidas sólo sobre ciertos conjuntos iniciales de números naturales determinadas por la disposición teleológica de $\mathrm{S}$ a responder ante la pregunta por la suma " $x+y$ " en los casos básicos. (Hago referencia a varias operaciones definidas por esa disposición teleológica debido al carácter gradual de la distinción entre casos básicos y restantes casos.) Para los propósitos de esta exposición, seguiré hablando de simplicidad, aunque el núcleo del asunto podría reconstruirse análogamente en términos de naturalidad o de semejanza natural.

Llamemos protosuma a cualquiera de esas operaciones definidas sólo sobre ciertos conjuntos iniciales de números naturales determinadas por la disposición teleológica de $\mathrm{S}$ concerniente a casos básicos. Otro sentido en que (iii) podría ser ambigua es el siguiente. Conforme a una forma sencilla de entender (iii), esta cláusula enunciaría ciertas relaciones entre operaciones matemáticas puramente abstractas: la operación más simple (o más natural) que generaliza a todos los números naturales la protosuma es la suma (más simple, en particular, que la parasuma); o bien: ninguna operación que generalice a todos 
los números naturales la protosuma es tan semejante a ella como la suma (tampoco lo es, en particular, la parasuma).

Por razones que no tendremos tiempo de examinar aquí, creo que esa lectura sencilla de (iii) no es la apropiada, pues — si la asumimos - el meollo del enigma sobre seguir una regla podría replantearse. Para resolver el enigma deben enunciarse relaciones entre entidades físicas del mundo natural (disposiciones teleológicas, según mi propuesta) y las operaciones matemáticas puramente abstractas. Conforme a la interpretación adecuada, es crucial entender que la cláusula (iii) enuncia relaciones entre las disposiciones teleológicas y las operaciones abstractas. Por decirlo así, la simplicidad o naturalidad intervendría doblemente: para fijar la(s) protosuma(s) a partir de la disposición teleológica y -según lo dicho en la interpretación sencilla de (iii) - para fijar la suma a partir de la(s) protosuma(s). Debo dejar los detalles de ese doble papel de la simplicidad en (iii) para otra ocasión. ${ }^{9}$

Kripke hace tres tipos de consideraciones críticas respecto a la posibilidad de invocar la simplicidad o la semejanza natural para ayudar a resolver el problema. Expone primero una objeción, relativa al tema de la simplicidad, justo después de su discusión sobre la hipótesis disposicional simple. Al acabar, desarrolla en una nota (n. 25) otras objeciones. Y más adelante (en la n. 45) considera y descarta la apelación a la semejanza natural. Veamos esos tres (grupos de) reflexiones.

La primera y la tercera de esas críticas son completamente impotentes contra la cláusula (iii) de (DW) y de (DT), pues combaten sendas estrategias para resolver el enigma que difieren sustancialmente de la estrategia propugnada por (iii). Conforme a una de esas estrategias, S se refiere a la suma porque ésta es una hipótesis más simple que la hipótesis alternativa (S se refiere a la parasuma). Kripke contesta, correctamente, que está en cuestión qué es referirse a la suma (y también, por tanto, qué es referirse a la parasuma). No es legítimo, pues, presuponer la existencia de dos hipótesis diferenciadas entre las cuales podamos decidir escoger la más simple. (Me parece errónea la contrarréplica de C. Wright (1984, n. 5) a esa observación

${ }^{9}$ Podría pensarse que no resulta problemático en absoluto cómo la disposición fija la(s) protosuma(s). Me parece un error (y no sólo por la diversidad de protosumas). Toda exposición del enigma sobre seguir una regla procede didácticamente focalizando las dificultades en ciertos aspectos y dando por sentado — por mor de la argumentación - otros aspectos. Pero el enigma en toda su plenitud es casi ubicuo en las relaciones de representación del mundo. 
de Kripke.) Pero la cláusula (iii) no habla de la simplicidad de hipótesis alguna. La simplicidad que invoca concierne a las relaciones entre una disposición y operaciones matemáticas abstractas.

La otra estrategia se basa en el supuesto acceso que tendría $\mathrm{S}$ (nuevamente aparece el requisito de la justificación racional) a la solución del enigma amparándose en el significado que $\mathrm{S}$ asigna a términos como "semejanza natural", "similar" o "absolutamente similar". Según la réplica de Kripke (también correcta, dada esa estrategia), cómo $\mathrm{S}$ hace referencia a la similitud no es menos problemático que cómo S hace referencia a la suma. Sus previos usos de "similar" admiten interpretaciones no estándar, tal y como sucede con sus previos usos de "suma". Esta réplica de Kripke es cercana al just more theory move esgrimido anticipadamente por Putnam contra los intentos de desbaratar su argumento teórico-modelista antirrealista invocando los hechos descritos por una teoría causal de la referencia (cfr. Putnam 1981, cap. 2). Y corre igual suerte: resulta efectiva si su destinatario pretende que son entidades lingüísticas las que resuelven el problema (el término "similar", o una teoría), pero completamente inocua frente a quien pretenda que es algo extralingüístico lo relevante (la similitud misma, en el caso que nos ocupa; ciertos hechos causales que una teoría aspira a describir, en el caso de la problemática abordada por Putnam).

La otras críticas de Kripke (expuestas brevemente en su n. 25) tienen mayor enjundia. Kripke menciona ahí una invocación a la simplicidad bien diferente a la que ha rechazado en el texto principal (la primera estrategia que acabo de comentar), y precisamente afín a lo propuesto en la cláusula (iii). La nota está integrada por tres párrafos. ${ }^{10}$ Kripke destaca tres dificultades interconectadas: en los dos primeros párrafos menciona problemas para definir el concepto de simplicidad pertinente; en la primera parte del último párrafo considera la hipótesis de que se hubiera definido el concepto de simplicidad y señala una dificultad concomitante; al final del último párrafo, se refiere nuevamente al aspecto justificatorio del enigma (el requisito epistémico internista), que afectaría a todo el asunto discutido en la nota. Adaptadas a nuestra discusión actual sobre (iii), podríamos verter en los siguientes términos esas tres dificultades.

\footnotetext{
${ }^{10}$ Un evaluador anónimo de Crítica me ha sugerido la conveniencia de exponer con mayor detalle mi interpretación de esta nota. Aunque no sé si coincidirá con mi atribución a Kripke de las tesis (K1) y (K2) que voy a señalar en el texto, y con mi posición respecto a dichas tesis.
} 
Quizá sea problemático hallar una apropiada caracterización de la noción de simplicidad según la cual es más simple correlacionar la disposición teleológica relevante de $\mathrm{S}$ con la suma que con otras operaciones matemáticas alternativas. Sin embargo, por sí mismo eso no invalida la propuesta (DW) ni la propuesta (DT), que estarían en peligro sólo si tuviéramos indicios de que la noción en cuestión es ininteligible o inaplicable. Tras esta crítica parece asomar nuevamente el requisito internista ajeno a $(\mathrm{DW})$ y $(\mathrm{DT})$ (la crítica sugeriría entonces que $\mathrm{S}$ debería disponer de una elucidación apropiada del concepto de simplicidad).

En segundo lugar, y más importante, Kripke (en el último párrafo de la nota) contempla la posibilidad de que se hubiera definido una medida de simplicidad y señala:

Se podría intentar [...] definir la función que quise decir como aquella que, de acuerdo con la medida de simplicidad, sigue el programa más simple aproximadamente compatible con mi estructura física. Supongamos que los fisiólogos del cerebro encontraran - para su sorpresaque en realidad tal medida de simplicidad nos conduce a un programa que calcula como función "+", no la adición, sino otra función distinta. ¿¿Mostraría esto que no quise decir adición mediante "+"? Y, sin embargo, a falta de un conocimiento detallado del cerebro (y de la hipotética medida de simplicidad), el descubrimiento fisiológico en cuestión no es en absoluto inconcebible. (1982, nota 25)

A continuación remite otra vez (antes de acabar el párrafo) al aspecto justificatorio del problema: no accedemos a esa hipotética medida de simplicidad cuando justificamos nuestra respuesta ante una suma.

En la medida en que estas críticas dependan de ese requisito epistémico internista sobre la justificación, podríamos dejarlas de lado recordando que (como señalé en la sección 4) es legítimo no presuponer dicho requisito. Pero el texto que acabo de citar apunta a una dificultad que requiere comentario. Según Kripke la siguiente hipótesis no puede descartarse a priori como algo inconcebible:

(H) La fisiología de nuestro cerebro (base física de la disposición teleológica relevante de S) es tal que, dada la mejor definición de simplicidad, resulta más simple correlacionar dicha disposición con una operación diferente a la suma.

Kripke da a entender dos tesis ulteriores: 
(K1) Aunque la hipótesis (H) fuera verdadera, cuando usé signos como "suma", "+" y "más" me estaba refiriendo a la suma; no me estaba refiriendo a esa otra operación diferente a la suma.

(K2) Las soluciones disposicionalistas (combinadas con la invocación del concepto de simplicidad) implican lo siguiente: si la hipótesis $(\mathrm{H})$ es verdadera, entonces cuando usé signos como "suma", "+" y "más" me estaba refiriendo a esa otra operación diferente a la suma.

A mi juicio, la tesis (K1) es correcta, pero (K2) es errónea. En la sección 4 hemos descartado un error semejante: que (DW) implicara $\left({ }^{*}\right)$ y $(* *)$. La réplica es análoga a lo dicho entonces. En primer lugar, $(\mathrm{H})$ tal vez sea imposible. Pero más importante: aunque $(\mathrm{H})$ fuera posible, no es legítimo sostener (K2). En virtud de principios desentrecomilladores (y algunos otros supuestos no cuestionados aquí) el consecuente de (Kl) es correcto ("cuando usé signos como 'suma', '+' y 'más' me estaba refiriendo a la suma") y por tanto es incorrecto el consecuente de la segunda parte de (K2). (La conjunción de $(\mathrm{H})$ con el disposicionalismo - combinado con la invocación del concepto de simplicidad - sólo refrenda enunciados contrafácticos subjuntivos, que no deben confundirse con el consecuente de la segunda parte de (K2), que está en tiempo verbal indicativo. El hecho de que en circunstancias contrafácticas muy diferentes hubiéramos usado "suma" para referirnos a algo diferente a la suma no es más extraño que el hecho de que en circunstancias contrafácticas diferentes hubiéramos usado "gato" para referirnos a animales diferentes a los gatos, pues podríamos haber llamado "gatos" a los perros, por ejemplo. Cfr. la sección 4.)

Quiero comentar brevemente una objeción a (DT) y a (DW) que, desde mi punto de vista, no coincide con ninguna de las críticas formuladas por Kripke. (La ha presentado Javier González de Prado ante una exposición oral de algunos de estos contenidos.) En cierto modo, va en dirección contraria a la sugerida por el texto de Kripke en relación con la hipótesis $(\mathrm{H})$. Consideremos un sujeto, S\#, similar al sujeto $\mathrm{S}^{*}$ descrito al final de la sección 4 , excepto porque aparentemente su discrepancia sistemática respecto a nosotros en modos de reaccionar no se produce ya en los casos básicos, sino sólo a partir de casos no básicos. (No se trata de alguien con disposiciones a errar por algún descuido sistemático.) La objeción consiste en lo siguiente. Intuitivamente deberíamos decir respecto a $\mathrm{S} \#$ lo mismo que debe decirse respecto a $\mathrm{S}^{*}$ : ese sujeto no está cometiendo errores respecto 
a la regla de sumar, sino que sigue correctamente una regla diferente, que es la regla a la que él hace referencia cuando usa "suma", "más", etc. Sin embargo, (DT) y (DW) parecerían implicar que S\# se refiere a la suma al usar esos signos, porque sus disposiciones a responder ante los casos básicos coinciden con las nuestras, y estamos suponiendo que, conforme a (iii), la operación más simple que extiende esas disposiciones es la suma.

A mi juicio, la mejor contrarréplica ante este problema requiere entender de forma relativizada el concepto de casos básicos. Las respuestas de $\mathrm{S} \#$ coinciden con las nuestras respecto a los casos que serían básicos para nosotros, o para muchos de nosotros. Sin embargo, según el modo en que la noción de caso básico, coordinada con la noción de simplicidad, contribuye a resolver el enigma sobre seguir una regla en una concepción disposicionalista, ésos no serían los casos básicos relevantes para el propio S\#. Los casos básicos o paradigmáticos relativamente a $\mathrm{S} \#$ constituyen un conjunto más amplio, en el cual se incluirían ya algunos casos ante los cuales las respuestas de $\mathrm{S} \#$ no coincidirían con las nuestras. De ese modo, no tenemos razones para pensar que la suma sea la operación matemática más simple que extiende a los casos no básicos para $\mathrm{S \#} \mathrm{las}$ disposiciones de $\mathrm{S} \#$ a responder ante casos básicos para $\mathrm{S} \#$.

En definitiva, no tenemos indicios de peso para pensar que soluciones disposicionalistas como (DW) y (DT) encuentren en el problema de la finitud una dificultad insuperable. Conforme a lo expuesto en la sección 4, esas soluciones disposicionalistas también pueden evitar el problema de la justificación racional, el problema de la normatividad y el problema de las disposiciones a errar. He tratado de cumplir así el propósito principal de esta contribución: proponer soluciones disposicionalistas resistentes ante las objeciones de Kripke contra la solución disposicional simple al enigma sobre seguir una regla. ${ }^{11}$

${ }^{11}$ Las ideas principales desarrolladas en este artículo se gestaron en dos grupos de lectura LOGOS, que coordiné en la Universidad de Barcelona, uno sobre el tema de seguir una regla (durante el curso 2009-2010) y otro sobre teleología y teleo-semántica (curso 2008-2009). He presentado versiones previas del texto en una conferencia invitada en la Universidad Autónoma de Madrid (el 27-2-2014) y en el XVII Congreso de AFRA (Santa Fé, Argentina, el 6-8-2015). Estoy en deuda con los participantes en esas reuniones, así como con otras personas que han leído el texto y también me han enviado comentarios y sugerencias. Mi agradecimiento, especialmente, a Marc Artiga, Ángel García, Javier González de Prado, Claudia Picazo, Daniel Quesada e Ignacio Vicario. En particular, he introducido correcciones y modificaciones relevantes tras las agudas observaciones críticas de J. González de Prado y C. Picazo. Financiación: Programa CONSOLIDER-INGENIO 2010, "Perspectival Thoughts and Facts" (CSD2009-00056), MICINN (Gobierno de España). 


\section{BIBLIOGRAFÍA}

Boghossian, Paul A., 1989, "The Rule-Following Considerations", Mind, vol. 98, no. 392, pp. 504-550. [http://links.jstor.org/sici?sici=00264423 \%28199001 \%292:99:393 \%3Cl:TROR \%3E2.0.CO;2-O]

Davidson, Donald, 1987, "Knowing One's Own Mind", Proceedings and Adresses of the American Philosophical Association, vol. 60, no. 3, pp. 441-458.

García-Carpintero, Manuel, 1996, Las palabras, las ideas y las cosas. Una presentación de la filosofía del lenguaje, Ariel, Barcelona.

Kripke, Saul, 1982, Wittgenstein on Rules and Private Languages. An Elementary Exposition, Harvard University Press, Cambridge. [Versión castellana (de la que procede la cita): Wittgenstein a propósito de reglas y lenguaje privado, Madrid: Tecnos, 2006.]

Lewis, David, 1983, "New Work for a Theory of Universals", Australasian Journal of Philosophy, vol. 61, no. 4, pp. 343-377.

Millikan Millikan, Ruth Garret, 1990, "Truth, Rules, Hoverflies, and the Kripke-Wittgenstein Paradox", Philosophical Review, vol. 99, no. 39, pp. 323-353.

Pérez Otero, Manuel, 2016, "Pluralismo plural en la concepción de Wittgenstein sobre seguir una regla", Daimon. Revista Internacional de Filosofía, vol. 67, pp. 73-81.

_- 2003, "Explicaciones funcionales", en Mario Casanueva y José Alberto Benítez (coordinadores), Representación y ciencia, M.A. Porrúa editor, México, pp. 65-84.

——, 2001, Aproximació a la filosofia del llenguatge, Edicions Universitat de Barcelona, Barcelona.

— 2000, "El argumento antiintelectualista de Wittgenstein sobre la comprensión del lenguaje", Theoria, vol. 15, no. 1, pp. 155-169.

—_, 1994, "Propiedades teleológicas y superveniencia", Éndoxa, no. 3, pp. 121-145.

Putnam, Hilary, 1981, Reason, Truth and History, Cambridge University Press, Cambridge.

Wittgenstein, Ludwig, 1967, Zettel. [Versión castellana: Zettel, UNAM, México, 1979.]

—_ 1958, The Blue and Brown Books, Oxford, Basil Blackwell. [Version castellana: Los cuadernos azul y marrón, Tecnos, Madrid, 1968.]

—_, 1953, Philosophische Untersuchungen. [Versión castellana: Investigaciones filosóficas, Crítica, Barcelona, 1988, edición bilingüe.]

Wright, Crispin, 1984, “Kripke's Account of the Argument against Private Language", Journal of Philosophy, vol. 81, no. 12, pp. 759-778.

Proyecto "Objetividad-subjetividad en el conocimiento y en la representación singular" (FFI2015-63892-P), MICINN. Grupo de investigación consolidado LOGOS (2014SGR81), AGAUR (gobierno catalán). 
Wright, Larry, 1976, Teleological Explanations: An Etiological Analysis of Goals and Functions, University of California Press, Berkeley and Los Ángeles. 168.

Recibido el 7 de marzo de 2015; revisado el 26 de octubre de 2015; aceptado el 19 de abril de 2016. 